\title{
Clinical evidence of traditional Chinese medicine for the prevention and treatment of chronic heart failure ${ }^{*}$
}

\author{
Shuo Zhang, Jiaying Wang, Hongcai Shang ${ }^{\#}$ \\ Evidence-Based Medicine Center of Tianjin University of Traditional Chinese Medicine, Tianjin, China \\ Email: "
}

Received 16 January 2013; revised 28 February 2013; accepted 20 March 2013

\begin{abstract}
Objective: To summarize clinical evidence of traditional Chinese medicine for the prevention and treatment of chronic heart failure systematically and comprehensively. Method: By retrieving in the database of VIP, WanFang, CNKI, PubMed, Cochrane Library, the qualifying results obtained in the databases above are summarized into the grading evidence including systematic reviews, randomized controlled trials, observational studies, case studies, basic research these five levels. Results: The numbers of the articles included are 12 systematic reviews, 413 randomized controlled trials, 68 observational studies, 15 case reports and 187 fundamental researches. Conclusion: It is concluded that basing on the clinical evidence of different levels, traditional Chinese medicine has prominent ability of preventing and treatment chronic heart failure.
\end{abstract}

Keywords: Traditional Chinese Medicine; Chronic Heart Failure; Clinical Evidence

\section{INTRODUCTION}

Chronic heart failure, also known as chronic-congestive heart failure, is defined as that chronic-primary cardiomyopathy and ventricular long-term pressure/volume overload cause the decrease of myocardial contractility which is not able to maintain cardiac output. It can be divided into two kinds, the left-right side of heart failure and heart failure. The common causes of this disease include rheumatic heart disease, hypertension, ischemic heart disease, myocarditis, aortic stenosis or regurgitation, ventricular septal defect, pulmonary heart disease, pulmonary valve stenosis and so forth. It is also the ultimate destination of the majority of cardiovascular disease and the main cause of death [1]. In Chinese medicine, heart failure can belongs to the area like palpitations, palpita-

\footnotetext{
"This article is supported by New Century Excellent Talent Project (NCET-09-0900).

${ }^{\#}$ Corresponding author.
}

tion, edema, asthma, phlegm, et al., and it is resulted from moisture within the stop and blood stasis attributed to non-normal transporting moisture and agitating-camping blood, due to the gradual decline of heart, spleen, kidney yang-energy. Deficiency of vital energy, blood stasis and water stop is a basic law of evolution in the pathogenesis of heart failure, and its intrinsic cause and ostensible appearance are deficiency of vital energy and blood stasis, water pan, respectively [2].

Clinical and basic research involving in prevention and cure of heart failure with traditional Chinese medicine have been reported greatly. However, there are few review related to the research about collecting and sorting these evidences. Accordingly, the advantages of traditional Chinese medicine in term of preventing and curing heart failure have not been fully confirmed. In this paper, we make the comprehensive collections of existing traditional Chinese medicine using in clinical evidence of heart failure prevention and through comprehensively analyzing the level of these evidence, the objective, reliable and comprehensive conclusions is obtained which can provide the reference for clinical decision-making.

\section{METHODS}

\subsection{Literature Search}

The retrieval was done in the databases of WanFang database, full-text database of Chinese Academic Journal (CNKI), Chinese Scientific Journals Database (VIP), Chinese Biomedical Medicine Database (CBM), PubMed and Cochrane Library. The retrieval strategy showed as follows (theme $=$ Chinese medicine OR proprietary Chinese medicines OR decoction) AND (theme $=$ cardiac failure OR heart failure), the time is until to March 2012. The references of retrieving literature are also traced, aiming at extending the range of relative research and reducing the undetected possibility.

\subsection{Inclusion Criterion}

The researching categories of inclusion include system- 
atic reviews, randomized controlled trials, observational studies, case studies, and experiments. Clinical studies in all cases are qualified to the diagnostic criteria for heart failure.

Method of Intervention: The treatment group, conventional therapy in randomized controlled trials + traditional Chinese medicine; the control group, conventional therapy.

Observational studies, case studies, basic research, conventional therapy + traditional Chinese medicine or traditional Chinese medicine.

Traditional Chinese medicine limited in oral medication or intravenous drugs, and not limited in specific dosage forms.

\subsection{Exclusion Criterion}

1) Not rigorous experimental design, not appropriate statistical methods, missing data in low-quality literature.

2) Similar and duplicated published literature.

\section{RESULTS}

Beginning of the seizure were found in 2356 literature, and filtered by NoteExpress software in duplicating versions, the remainder are the 1574 literature; by excluding the non-relative title, abstract, obviously literature (533), the remaining are 1041 literature; by learning the content of full text, further excluding are done to the articles which are not quailed to the inclusion criteria, eventually including in the 695 literature.

\subsection{System Evaluation}

Systematic reviews include in the 12 traditional Chinese medicines to prevent cardiac failure [3-14], it contains a total of 248 clinical studies, and subjects were 16,181 people. Drugs used in traditional Chinese medicine injection are the most part. There are 5 papers in simple usage of Chinese medicine injection (41.67\%); the number of paper in Shengmai injection and Huangqi injection are both 2; 2 paper are in Chinese herbal compound; Shenfu injection, Qiliqiangxin capsule and Lingguizhugan soup are in 3 articles, respectively.

Since quality assessment reference standard is not fully consistent, and the description of the results is also different, it is hard to summarize their characteristics in detail. However, overall, the qualities of the literature included are lower.

All studies are employed with Meta-analysis way and then the results of efficacy evaluation are obtained from that.

Observed rate $=$ the literature number mentioned in the presence-absence adverse reactions occurred/total number of included studies.

- 3 Literatures did not mention the security observed rate of the original clinical data in this study included 12 articles.

- 9 articles are reported in adverse reactions, including adverse reactions in 8 literatures, two of which did not report the observed rate; in remainder, the highest observed rate was $68.63 \%$; overall, the adverse reactions of traditional Chinese medicine is a gastrointestinal discomfort, less occurring and not serious. No adverse is in 1 article (see Table 1).

\subsection{Randomized-Controlled Trials}

There are a total of 413 articles retrieved. Overall, the randomized controlled trials of traditional Chinese medicine prevention and treatment of heart failure presents an increasing trend (Figure 1): the use of Chinese medicine formulations decoction is the most (275 articles), and then the other formulations are injection (69 articles), proprietary Chinese medicines in capsules (19 articles), granules (19 articles), powders (10 articles), pills (10 articles), tablets (5 articles), ointment (3 articles), Dan agent 2 articles, as a total of 68 articles. Drug use in observation group are mostly in the form of Chinese medicine plus Western medicine, in which there are decoction + Western medicine (268 articles), Chinese patent medicine + Western medicine (66 articles), Chinese medicine injections + Western medicine (64 articles), only 12 articles involve in separating use of traditional Chinese medicine treatment. Drugs used include oral proprietary 42 species and 21 kinds of Chinese medicine injection; the decoctions used are mostly self decoction or existing decoction adding/subtracting, and since a wide variety of traditional Chinese medicine are involved, detailed statistics are not given (see Table 2).

In retrieved Randomized-controlled trials of Chinese medicine for prevention and treatment of heart failure, the treatments are the vast majority of conventional western medicine treatment on the basis of the use of Chinese medicine, and then compare with purely conventional western medicine treatment.

\subsection{Observational Studies}

A total of 68 articles are retrieved. Chinese medicine formulations are mostly used in decoction (53 articles); there are Chinese medicine injections (11 articles), proprietary Chinese medicines (4 articles), powder (2 articles), and paste (1 article). The major treatment is the combination of Chinese traditional and Western medicine: decoction + Western medicine (33 articles), Chinese medicine injections + Western medicine (10 articles), Chinese patent medicines + Western medicine (4 articles), the use of traditional Chinese medicine only (24 articles). Types of Chinese medicine used: oral proprietary 7 kinds, Chinese medicine injections 8 kinds; the decoctions used are mostly self decoction or existing 
Table 1. The results of the evaluation of traditional Chinese medicine prevention and treatment of heart failure system.

\begin{tabular}{|c|c|c|c|c|c|c|c|}
\hline \multirow[b]{2}{*}{ Research } & \multirow[b]{2}{*}{ Interventions } & \multirow{2}{*}{$\begin{array}{l}\text { Included } \\
\text { Studies }\end{array}$} & \multirow[b]{2}{*}{ Subjects } & \multirow{2}{*}{$\begin{array}{l}\text { Quality } \\
\text { Evaluate }\end{array}$} & \multirow{2}{*}{$\begin{array}{l}\text { Efficacy } \\
\text { Positive Results }\end{array}$} & \multicolumn{2}{|c|}{ Security } \\
\hline & & & & & & $\begin{array}{l}\text { Observed } \\
\text { Rate }\end{array}$ & $\begin{array}{c}\text { Bad } \\
\text { Reaction }\end{array}$ \\
\hline $\begin{array}{l}\text { Wen, Z.H. } \\
2006 \text { [3] }\end{array}$ & $\begin{array}{l}\text { Traditional Chinese } \\
\text { medicine \#1 }\end{array}$ & 51 & Unknown & $\begin{array}{l}\text { Jadad } \\
\text { high-quality } 1 \\
\text { low-quality the remaining }\end{array}$ & Clinical efficacy & $68.63 \%$ & $\begin{array}{l}\text { yes } \\
\# 2\end{array}$ \\
\hline $\begin{array}{l}\text { Bin, X.F. } \\
2009[4]\end{array}$ & Shenfu injection & 8 & 875 & $\begin{array}{l}\text { Jadad } \\
\text { low-quality }\end{array}$ & Clinical efficacy & Unknown & Not mentioned \\
\hline $\begin{array}{l}\text { Luo, M.X. } \\
2009[5]\end{array}$ & Shengmai injection & 19 & 1440 & C-level & $\begin{array}{l}\text { Cardiac function classification, } \\
\text { Cardiac function indicators }\end{array}$ & $52.63 \%$ & $\begin{array}{l}\text { yes } \\
\# 3\end{array}$ \\
\hline $\begin{array}{l}\text { Wang, L.X. } \\
2009 \text { [6] }\end{array}$ & Huangqi injection & 11 & 1044 & $\begin{array}{l}\text { Jadad } \\
\text { high-quality } 1 \\
\text { low-quality the remaining }\end{array}$ & $\begin{array}{l}\text { Clinical efficacy, } \\
\text { Left ventricular ejection fraction }\end{array}$ & Unknown & $\begin{array}{l}\text { yes } \\
\# 4\end{array}$ \\
\hline $\begin{array}{l}\text { Xu, W.B. } \\
2010[7]\end{array}$ & Yiqi huoxue fufang & 17 & 1442 & C-level & $\begin{array}{l}\text { Cardiac function, } \\
\text { Left ventricular ejection fraction }\end{array}$ & $5.88 \%$ & no \\
\hline $\begin{array}{l}\text { Duan, F. } \\
2010[8]\end{array}$ & $\begin{array}{l}\text { Yiqi huoxue } \\
\text { Traditional Chinese } \\
\text { medicine }\end{array}$ & 16 & 1286 & $\begin{array}{l}\text { Jadad } \\
\text { high-quality } 1 \\
\text { low-quality the remaining }\end{array}$ & $\begin{array}{l}\text { Clinical efficacy, } \\
\text { Left ventricular ejection fraction }\end{array}$ & Unknown & $\begin{array}{l}\text { yes } \\
\# 5\end{array}$ \\
\hline $\begin{array}{l}\text { Liu, C.X. } \\
2010 \text { [9] }\end{array}$ & $\begin{array}{l}\text { Qiliqiangxin } \\
\text { Capsule }\end{array}$ & 7 & 403 & B-level 1, C-level & $\begin{array}{l}\text { Cardiac function classification, } \\
6 \text { min walk test, } \\
\text { Left ventricular ejection fraction, } \\
\text { LVEDD, Quality of life }\end{array}$ & $57.14 \%$ & $\begin{array}{l}\text { yes } \\
\# 6\end{array}$ \\
\hline $\begin{array}{l}\text { Wang, J. } \\
2011[10]\end{array}$ & Oral medicine \#7 & 60 & 5344 & $\begin{array}{l}\text { Jadad } \\
\text { High-quality } 8 \\
\text { low-quality the remaining }\end{array}$ & $\begin{array}{l}\text { Left ventricular ejection fraction, } \\
6 \text { min walk test, Adverse reactions }\end{array}$ & $16.67 \%$ & $\begin{array}{l}\text { yes } \\
\# 8\end{array}$ \\
\hline $\begin{array}{l}\text { Zhu, L.L. } \\
2011[11]\end{array}$ & $\begin{array}{l}\text { Yang water herbal } \\
\text { compound\#9 }\end{array}$ & 31 & 2414 & C-level & $\begin{array}{l}\text { Clinical efficacy, BNP, } \\
6 \text { min walk test, } \\
\text { Cardiac function indicators }\end{array}$ & $32.3 \%$ & $\begin{array}{l}\text { yes } \\
\# 10\end{array}$ \\
\hline $\begin{array}{l}\text { Chen, J. } \\
2011 \text { [12] }\end{array}$ & Shengmai injection & 14 & 942 & C-level & Clinical efficacy & $42.86 \%$ & $\begin{array}{l}\text { yes } \\
\# 11\end{array}$ \\
\hline $\begin{array}{l}\text { Qiu, M.L. } \\
2011[13]\end{array}$ & Lingguizhugan soup & 4 & 280 & C-level & $\begin{array}{l}\text { Clinical efficacy } \\
\text { Left ventricular ejection fraction }\end{array}$ & Unknown & Not mentioned \\
\hline $\begin{array}{l}\text { Wen, Z.H. } \\
2011 \text { [14] }\end{array}$ & Huangqi injection & 10 & 711 & $\begin{array}{l}\text { Jadad } \\
\text { low-quality }\end{array}$ & $\begin{array}{l}\text { Clinical efficacy } \\
\text { Left ventricular ejection fraction }\end{array}$ & Unknown & Not mentioned \\
\hline
\end{tabular}

Tip: \#1 = Shengmai, Shenmai, Shenfu, Huangqi injection and Shengmai powder and decoction; \#2 = 11 articles reported 38 cases of side effect in treatment group; \#3 = 6 articles reported slight adverse effect; \#4 = no obvious side effect except a case of vomiting in treatment and 12 cases of headache and dizzy, 10 cases of hot flash, five cases of cough and 2 cases of nausea; \#5 = In the control group, 1 article appeared digitalis poisoning and water salt electrolyte disorder, and 12 cases of dizzy, 2 cases of nausea and 3 cases of severe heart event; \#6 = dizzy, cough, epigastrium pain, gasteremphraxis; \#7 = the single herb, the compound decoction and the Chinese patent drugs; \#8 = no side effect was reported in six articles, and 4 articles reported the slight abdominal discomfort; \#9 = the orally taken formula consisted of warming yangqi and diuresis herbs; $\# 10=4$ articles reported the side effect and three of them pointed that it was caused by other drugs and one article reported the adverse effect in the controlled group; \#11 = 2 articles reported the dizzy and headache.

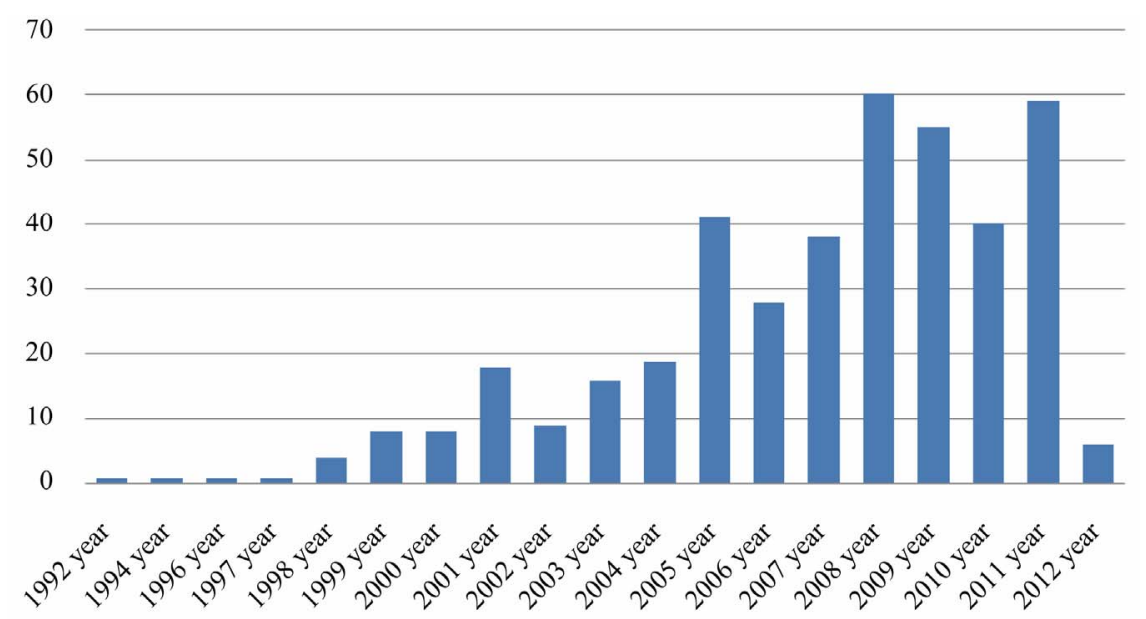

Figure 1. The amount of the literature. 
Table 2. Chinese patent medicine use of a variety of clinical studies.

\begin{tabular}{|c|c|c|c|}
\hline Type of research & Category & Traditional Chinese medicine & Articles. \\
\hline \multirow{14}{*}{$\begin{array}{l}\text { Randomized controlled } \\
\text { trials }\end{array}$} & \multirow{8}{*}{ injection } & Shengmai injection & 20 \\
\hline & & Huangqi injection & 16 \\
\hline & & Shenmai injection & 15 \\
\hline & & Shenfu injection & 12 \\
\hline & & Danshen injection & 4 \\
\hline & & Xinmailong injection, Fufangdanshen injection, Gegensu injection & 3 \\
\hline & & Danhong injection, Ciwujia injection & 2 \\
\hline & & $\begin{array}{l}\text { Xiangdan, Honghua, Mailuoning, Guanxinning, Gaolishen, Dengzhanxixin, } \\
\text { Dengzhanhuasu, Danshenduofensuan, Chuanxiongqin, Shenqifuzheng, Shenfuqi }\end{array}$ & 1 \\
\hline & \multirow{6}{*}{ Oral Liquid } & Qiliqiangxin Capsule & 8 \\
\hline & & Shengmai Powder & 5 \\
\hline & & Heart-Protecting Musk Pill, Qiangxintongmai Granules, Manshuailing Oral Liquid & 4 \\
\hline & & $\begin{array}{l}\text { Wenxin Granules, Jaweishenfu Granules, Fufangzhenwu Chongji, Wuling Powder, } \\
\text { Yixin Paste }\end{array}$ & 3 \\
\hline & & $\begin{array}{l}\text { Janxin Granules, Qiangxin Granules, Nuanxin Capsule, Shensongyangxin Capsule, } \\
\text { Baoxinkang Tablets, Kangdaxin Oral Liquid, Wenyangjianxinling Oral Liquid }\end{array}$ & 2 \\
\hline & & $\begin{array}{l}\text { Zhenyuan Capsule, Tongmaiqiangxin Capsule, Qiangxinli Capsule, Kangxinli Capsule, } \\
\text { Yixinshu Capsule, Xuezhikang Capsule, Suixin Capsule, Yiqishuxin Pill, Yixintai Pill, } \\
\text { Qiangxin Dripping Pill, Kangxinshuai Pill, Xinlishukang Pill, } \\
\text { A strong heart Tongmai Pellets, Xinshengmai Tablets, Xinshuaikang Tablets, } \\
\text { Fufanghuangliansu Tablets, Qiangxin Oral Liquid, Qiangxinkang, } \\
\text { Guanxinkang Oral Liquid, Buxinqi Oral Liquid, Pd extract, Kangxin Powder, } \\
\text { Sengmaiqiangxin Powder, Tianxiangdan, Huangqi Granules, } \\
\text { Hongguixinlikang Chongji }\end{array}$ & 1 \\
\hline \multirow{4}{*}{$\begin{array}{l}\text { Observational } \\
\text { Research }\end{array}$} & \multirow{3}{*}{ injection } & Shengmai, Shenfu, Danshen, Huangqi & 3 \\
\hline & & Fufangdanshen, Shenmai & 2 \\
\hline & & Mailuoning, Ciwujia & 1 \\
\hline & Oral Liquid & $\begin{array}{l}\text { Shengmai Powder, Wuling Powder, Wupi Powder, Huangliansu Tablets, Xuefuzuyu } \\
\text { Oral Liquid, Buchang wenxinkeli, Evergreen cream }\end{array}$ & 1 \\
\hline \multirow{2}{*}{ Medical record study } & injection & Honghua, Huangqi, Shengmai & 1 \\
\hline & Oral Liquid & Qubi Tongluo Paste & 1 \\
\hline
\end{tabular}

decoction adding/subtracting, and since a wide variety of traditional Chinese medicine are involved, detailed statistics are not given (see Table 2).

Observational studies of existing Chinese medicine prevention and treatment of heart failure are mostly using the treatment method of combination of Chinese traditional and Western medicine, the acute phase of heart failure are more with traditional Chinese medicine injection, and chronic heart failure is more dialectical usage with the decoction and proprietary Chinese medicines.

\subsection{Medical Record Studies}

A total of 15 documents are retrieved and they were decoction treatment: Chinese medicine injections (2 articles), proprietary Chinese medicines (1 article, available as an ointment). Treatment method: decoction + Western medicine (8 articles), decoction + Chinese medicine injections + Western medicine (2 articles), decoction +
Chinese patent medicines + Western medicine ( 1 article), traditional Chinese medicine only (7 articles). Types of traditional Chinese medicine: oral proprietary 1 kind, Chinese medicine injections 3 kinds. The decoctions used are mostly self decoction or existing decoction adding/subtracting, and since a wide variety of traditional Chinese medicine are involved, detailed statistics are not given (see Table 2).

In the early medical records of Chinese medicine prevention and treatment of heart failure, most of the traditional Chinese medicine (soups, Chinese patent medicines and injection) are used alone to cure chronic heart failure, however, recent literature involve in using treatment method of combination of Chinese traditional and Western medicine to cure this disease.

\subsection{Basic Research}

A total of 187 in the basic research of traditional Chinese 
Table 3. Basic research of Chinese medicine usage.

\begin{tabular}{lccl}
\hline Category & Articles & Total & Typical representative \\
\hline Herbal Monomer & 15 & 10 & Astragalus polysaccharides, Tanshinone \\
Chinese medicine injection & 4 & 4 & Danshen injection, Huangqi injection \\
Proprietary Chinese medicines & 65 & 32 & Wenxin Granules, Qiliqiangxin Capsule \\
Jingfang & 4 & 2 & Lingguizhugan soup, Zhen Wu Tang \\
Total & 88 & 48 & - \\
\hline
\end{tabular}

medicine prevention and treatment of chronic heart failure are retrieved. There are 42 papers in the study included the experiments in vitro. Animal models mostly are rats (165 articles), rabbits (18 articles), guinea pigs, suckling mice, dogs and cats (1 article to each). Basic researches of Chinese medicine used are mostly self decoction or existing Chinese medicine, a total of 99 articles. In these articles, types of Chinese medicine involved are the more part, therefore no detailed statistics are done. Chinese patent medicines are the second in the study, 65 articles and 32 kinds in details. Chinese medicine monomer and existing Chinese medicine have also been used, but they just occupy a smaller proportion (see Table 3).

Existing basic research results demonstrate that traditional Chinese medicine can affect prominently in the prevention and treatment of heart failure through the aspects of multi-channel, multi-level, multi-target. The mechanism involves in the following aspects: regulation of inflammatory cytokines, inhibition of cardiomyocyte apoptosis, improve cardiac contractility, diastolic function, reduce organ congestion, correct the neuroendocrine hormone releasing imbalance, inhibition of extra-cellular matrix remodeling, anti-myocardial fibrosis, anti-ventricular remodeling.

\section{CONCLUSIONS}

The results of system evaluation show that the advantages of chronic heart failure treatment by traditional Chinese medicine therapy are as following: decreasing mortality, reducing incidence, increasing ejection fraction, controlling the ventricular remodeling (shrink and diastole last phase volume), improving cardiac function and enhancing the overall curative effect.

The study results of the randomized controlled trials show that it has more advantages to use the combination of traditional Chinese medicine and western medicine to treatment chronic cardiac failure compared with western medicine only. The therapeutic effect of chronic cardiac failure and side effect are all improved.

The results of observational studies and case studies show that using traditional Chinese medicine has good effect on treating chronic heart failure without obvious side effect.
The existing fundamental research results show that traditional Chinese medicine can treat chronic heart failure through many methods, levels and target points. The mechanism include regulating and controlling inflammatory cytokines, restraining death of cardiac muscle cell, improving the shrink and diastoling function, reducing hyperemia of heart, rectifying the unbalance releasing condition of neuroendocrine, restraining the reconstruction of extracellular matrix, resisting myocardial fibrosis and ventricular remodeling. Case and observational studies show that the advantage of treating chronic heart failure by Chinese medicine is improving clinical symptoms and prognosis. The system evaluation further affirms the advantage of Chinese therapy.

Papers searched show that present clinical study is mainly randomized controlled trial. Although the randomized controlled trial is a high level clinical evidence, the low quality of study design and not entire report may influent the results of this paper.

\section{REFERENCES}

[1] Ye, R.G. (2004) Internal medicine. 6th Edition, People’s Medical Publishing House, Beijing.

[2] Gu, D.F., Huang, G.Y. and He, J. (2003) China’s heart failure epidemiological survey and prevalence. Chinese Journal of Cardiology, 31, 3-6.

[3] Wen, Z.H. (2006) Meta-analysis of randomized controlled trials of traditional Chinese medicine treatment for chronic heart failure. The First National TCM of the Professional Committee of China Association of Integrative Medicine Evidence-Based Medicine, Integrative Medicine Symposium Proceedings of Evidence-Based Medicine, Guangzhou, 26-28 November 2006, 292-302.

[4] Bin, X.F. and Guo, Z.H. (2010) Shenfu injection for the treatment of congestive heart failure: A systematic review and Meta-analysis. JGCTCM, 32, 76-79.

[5] Luo, M.X. (2009) Clinical effect of Shengmai injection on chronic heart failure: A meta-analysis. Master's Thesis, Southern Medical University, Guangzhou.

[6] Wang, L.X., Du, W.X. and Zhu, M.D. (2009) A systematic review on Huangqi injection for chronic heart failur. Chinese Journal of Evidence-Based Cardiovascular Medicine, 1, 78-81.

[7] Xu, W.B. (2010) Compound traditional Chinese medieine with Qi-supplementing and blood activating for chronic 
heart failure on systematic reviews of randomised controlled trials. Master's Thesis, Chengdu University of Traditional Chinese Medicine, Chengdu.

[8] Duan, F. and Gao, P.Y. (2010) Meta analysis: Yiqihuoxue formulas are effective on treating chronic heart failure. Research of Integrated Traditional Chinese and Western Medicin, 2, 238-242.

[9] Liu, C.X., Mao J.Y. and Wang X.L. (2010) A systematic review for Qili Qiangxin Capsule for chronic heart failure. Chinese Traditional Patent Medicine, 32, 539-544.

[10] Wang, J., Chen, C. and Zhang, P. (2012) Systematic review and Meta-analysis of randomized clincal trials of oral Chinese medicine for treatment on chronic heart failure. China Journal of Traditional Chinese Medicine and Pharmacy, 26, 2830-2840.

[11] Zhu, L.L. (2011) Traditional Chinese medieine with warming yang for diuresis for patients with chronie heart failure: A systematic review. Master's Thesis, Chengdu University of Traditional Chinese Medicine, Chengdu.

[12] Chen, J., Luo, M.X. and Zheng, Q. (2011) Effect of Shengmai injection on patients with chronic heart failure: A Meta-analysis. Chinese Journal of Information on TCM, 18, 25-28.

[13] Qiu, M.L., Mao, J.Y. and Wang, J.Y. (2011) A metaanalysis: Effect of linggui zhugan or added formula on chronic heart failure. Chinese Journal of Experimental Traditional Medical Formulae, 17, 243-247.

[14] Wen, Z.H., Nong, Y.B. and Pan, C.X. (2011) Astragalus injection for the treatment of chronic heart failure: A systematic review and Meta-analysis. Chinese Journal of Integrative Medicine on Cardio-/Cerebrovascular Disease, $\mathbf{9}$, 770-772. 